Supplement of Biogeosciences, 11, 5503-5519, 2014

http://www.biogeosciences.net/11/5503/2014/

doi:10.5194/bg-11-5503-2014-supplement

(C) Author(s) 2014. CC Attribution 3.0 License.

(c) (i)

Supplement of

\title{
Biogeophysical feedbacks enhance the Arctic terrestrial carbon sink in regional Earth system dynamics
}

W. Zhang et al.

Correspondence to: W. Zhang (zhang_wenxin2005@hotmail.com) 


\section{Index:}

Fig. S1. Arctic tundra domain (red) (McGuire et al., 2012) and the CORDEX-Arctic domain (red and yellow).

Fig. S2. Scatter plot comparing the simulated NPP flux in the RCA-GUESS simulation and the NPP flux validation datasets (EMDI (Olson et al., 2013a), BAZ (Denissenko et al., 2013), GPPDI_1 (Olson et al., 2013b), GPPDI_2 (Zheng et al., 2013), BOREAL (Gower et al., 2012)).

Fig. S3. The inter-annual variation of NEE anomalies for the RCA-GUESS feedback and non-feedback runs, process-based models (LPJ-GUESS WHyMe, ORCHIDEE, TCF and TEM6) and the ensemble mean of inversion models for the period 1990-2009, averaged over the Arctic tundra domain defined by McGuire et al. (2012).

Fig. S4. The cumulative NEE simulated by the RCA-GUESS feedback and non-feedback runs for the CORDEX-Arctic domain compared to the stand-alone simulations of LPJGUESS forced by the CMIP5 products for the land areas above $60^{\circ} \mathrm{N}$ (Ahlström et al., 2012) (dark color: forcing bias corrected; light color: forcing bias uncorrected).

Fig. S5. The effects of biogeophysical feedbacks on the sum of net short-wave and net long-wave radiation fluxes $\left(\mathrm{W} \mathrm{m}^{-2}\right.$ ) in the summer (June, July and August) for 2071-2100.

Table S1. Parameters of PFTs used in RCA-GUESS (BNE-boreal needle-leaved evergreen forests, BINE-boreal shade-intolerant needle-leaved evergreen trees, BNSboreal needle-leaved deciduous trees, TBS-temperate broad-leaved deciduous trees, IBSboreal shade-intolerant deciduous forests, GRS-C3 grass).

Table S2. The key numerical equations used for the RCA-GUESS coupling processes and the quantification of biogeophysical feedback impacts on vegetation change.

Table S3. Aggregation of the International Satellite Land Surface Climatology Project (ISLSCP) II Potential Natural Vegetation (PNV) Cover dataset and the Kaplan PNV dataset to five vegetated classes for comparison with plant functional types in RCAGUESS. 


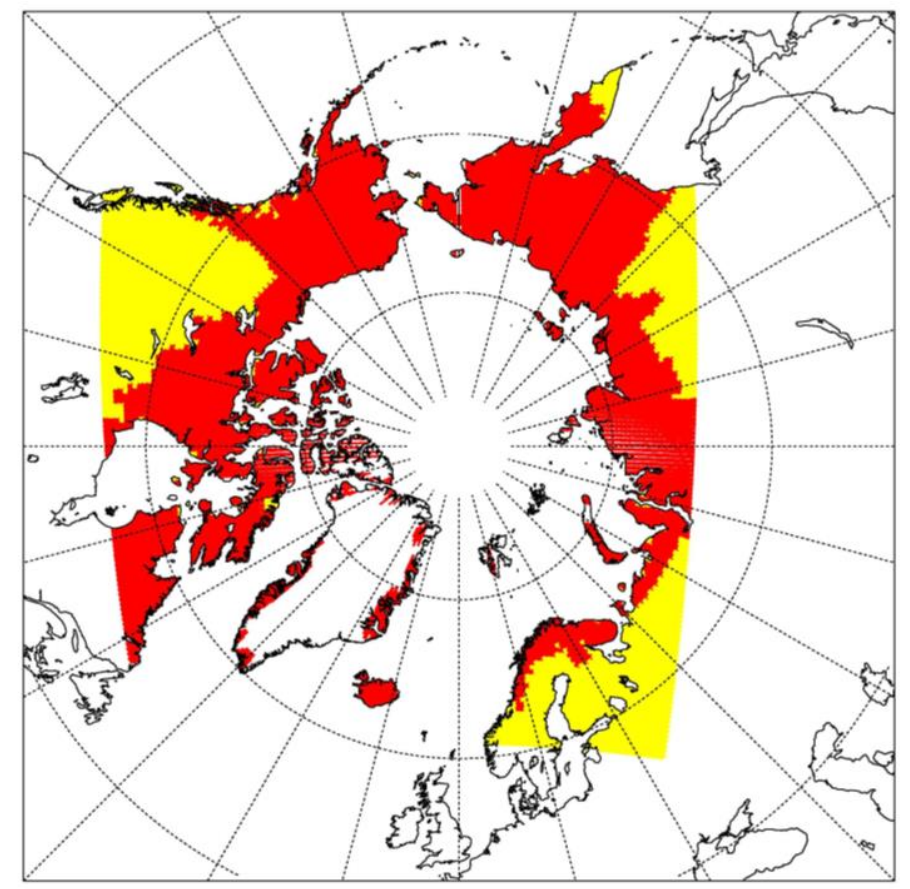

Fig. S1. The Arctic tundra domain (red) (McGuire et al., 2012) and the CORDEX-Arctic domain (red and yellow).

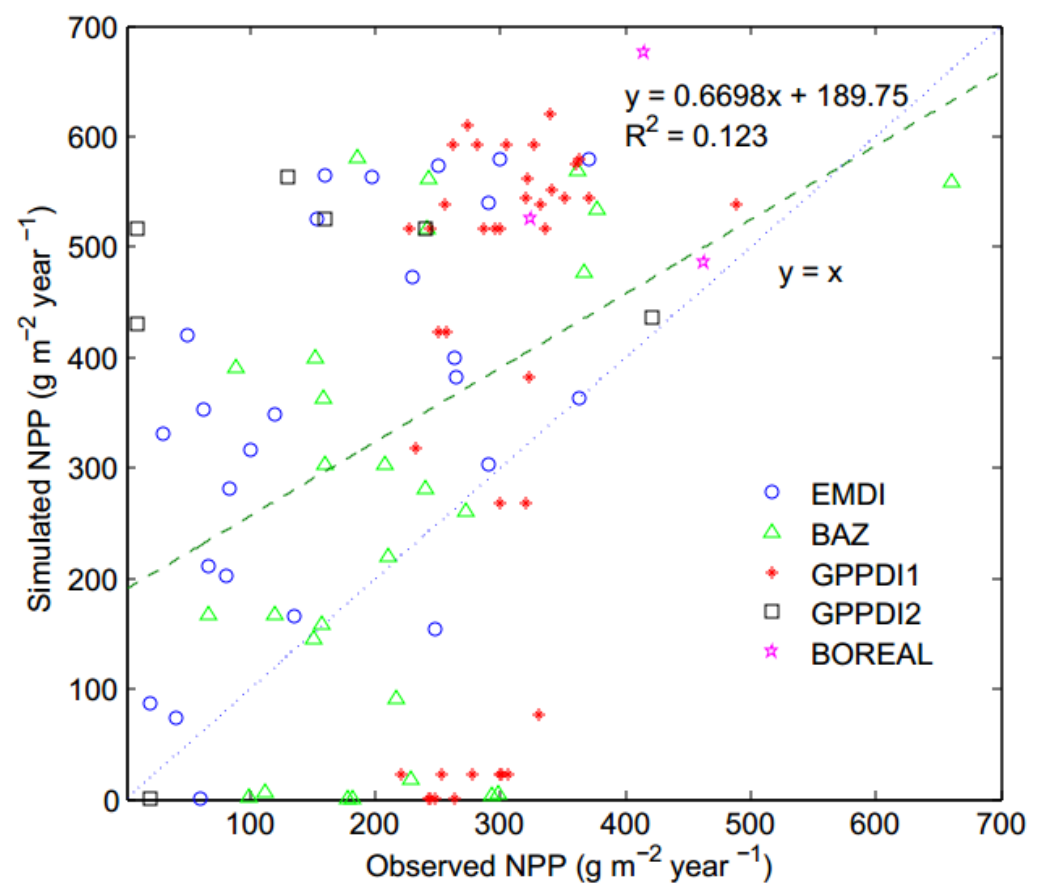

Fig. S2. Scatter plot comparing the simulated NPP flux in the RCA-GUESS simulation and the NPP flux validation datasets (EMDI (Olson et al., 2013a), BAZ (Denissenko et al., 2013), GPPDI_1 (Olson et al., 2013b), GPPDI_2 (Zheng et al., 2013), BOREAL (Gower et al., 2012)). 


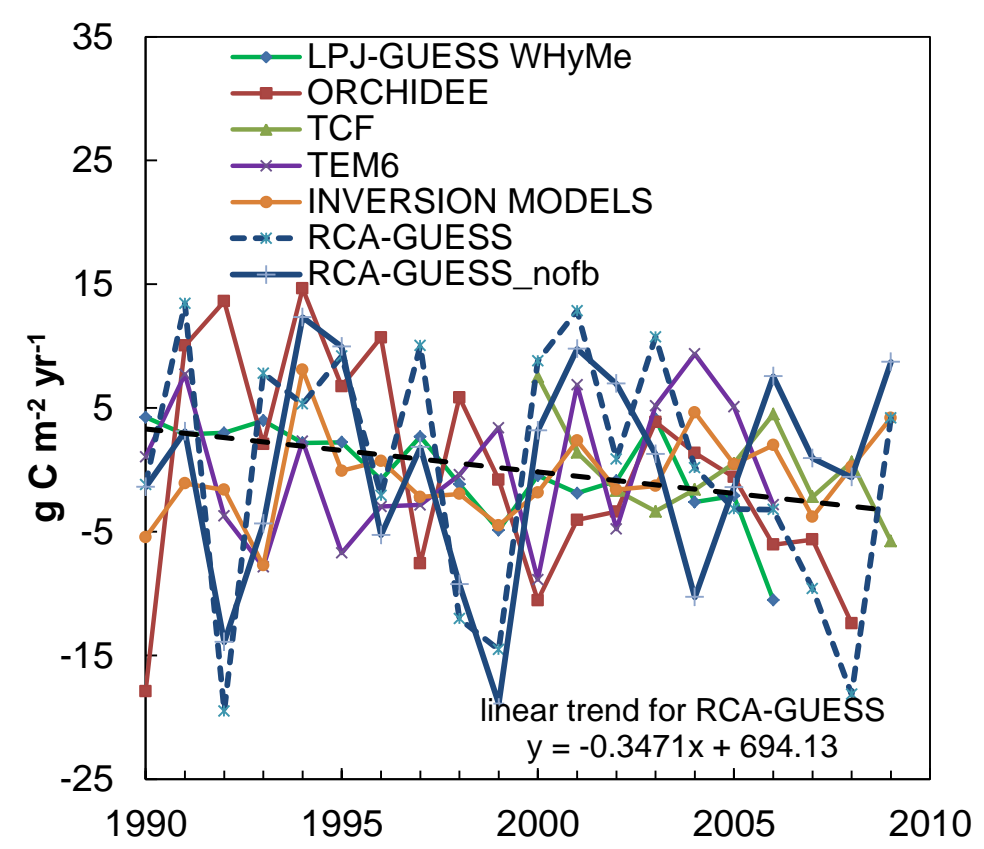

Fig. S3. The inter-annual variation of NEE anomalies for the RCA-GUESS feedback and non-feedback runs, process-based models (LPJ-GUESS WHyMe, ORCHIDEE, TCF, TEM6) and the ensemble mean of inversion models for the period 1990-2009 averaged over the Arctic tundra domain defined by McGuire et al. (2012).

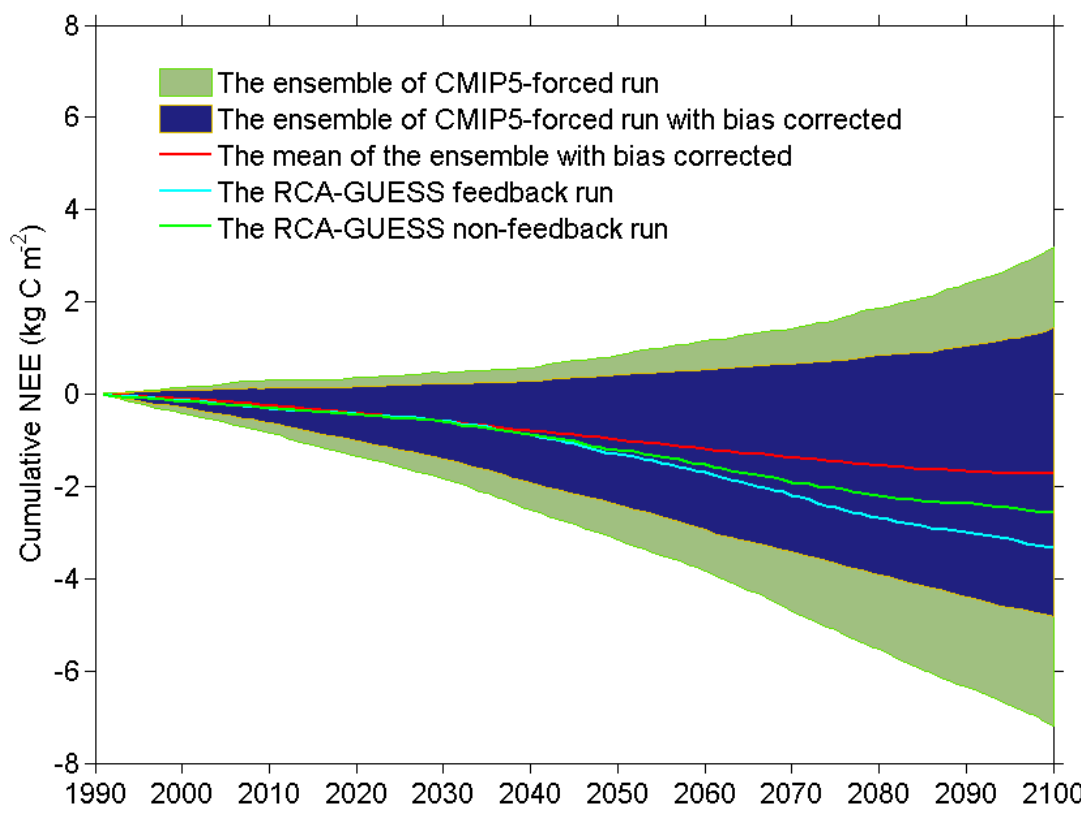

Fig. S4. The cumulative NEE simulated by the RCA-GUESS feedback and non-feedback runs for the CORDEX-Arctic domain compared to the stand-alone simulations of LPJGUESS forced by the CMIP5 products for the land areas above $60^{\circ} \mathrm{N}$ (Ahlström et al., 2012b) (dark color: forcing bias corrected; light color: forcing bias uncorrected). 


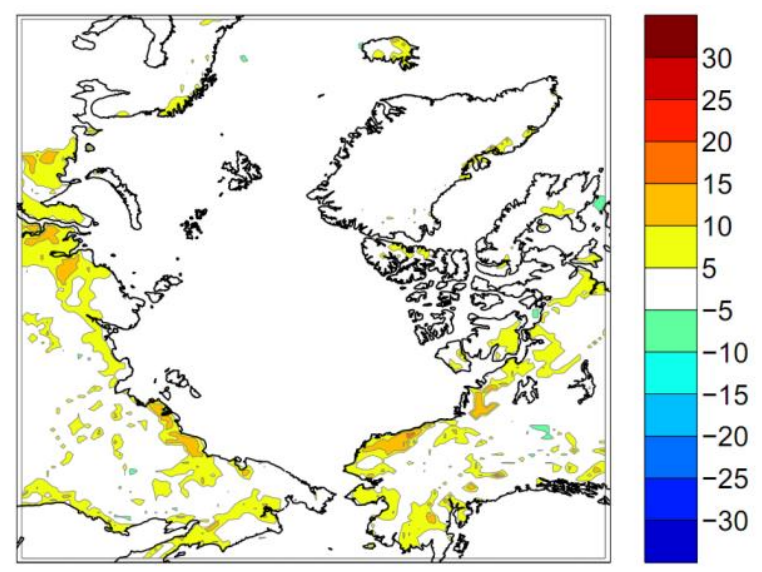

Fig. S5. The effects of biogeophysical feedbacks on the sum of net short-wave and net long-wave radiation fluxes $\left(\mathrm{W} \mathrm{m}^{-2}\right)$ in the summer (June, July and August) for 2071-2100. 
Table S1. Parameters of PFTs used in RCA-GUESS (BNE-boreal needle-leaved evergreen trees, BINE-boreal shade-intolerant needle-leaved evergreen trees, BNS-boreal needle-leaved deciduous trees, TBS-temperate broad-leaved deciduous trees, IBS-boreal shade-intolerant deciduous trees, GRS-C3 grass).

\begin{tabular}{|c|c|c|c|c|c|c|c|}
\hline Parameter & Description (Units) & BNE & BINE & BNS & TBS & IBS & GRS \\
\hline alphar & Recruitment shape parameter & 3 & 10 & 3 & 3 & 10 & - \\
\hline bulk_dens & Bulk density $\left(\mathrm{kg} \mathrm{m}^{-3}\right)$ & 20 & 20 & 20 & 20 & 20 & 2 \\
\hline crownarea_max & Max. tree crown area $\left(\mathrm{m}^{2}\right)$ & 50 & 50 & 50 & 50 & 50 & - \\
\hline cton_leaf & Leaf C:N mass ratio (-) & 29 & 29 & 29 & 29 & 29 & 29 \\
\hline cton_root & Root C:N mass ratio (-) & 29 & 29 & 29 & 29 & 29 & 29 \\
\hline cton_sap & Sapwood C:N mass ratio (-) & 330 & 330 & 330 & 330 & 330 & - \\
\hline est_max & Max. sapling establishment rate (individual $\mathrm{m}^{-2} \mathrm{yr}^{-1}$ ) & 0.05 & 0.2 & 0.05 & 0.05 & 0.2 & - \\
\hline fireresist & Fire resistance $(0-1)$ & 0.3 & 0.3 & 0.3 & 0.1 & 0.1 & 0.5 \\
\hline gdd5min_est & Min. GDD5 for establishment $\left({ }^{\circ} \mathrm{C}\right.$ day) & 500 & 500 & 350 & 1100 & 300 & 0 \\
\hline gmin & Min. canopy conductance $\left(\mathrm{mm} \mathrm{s}^{-1}\right)$ & 0.3 & 0.3 & 0.3 & 0.5 & 0.5 & 0.5 \\
\hline greff_min & Threshold for growth suppression mortality $\left(\mathrm{kg} \mathrm{m}^{-2} \mathrm{yr}^{-1}\right)$ & 0.04 & 0.08 & 0.04 & 0.04 & 0.08 & - \\
\hline intc & Interception coefficient & 0.06 & 0.06 & 0.06 & 0.02 & 0.02 & 0.01 \\
\hline kest_repr & Constant in equation for budburst chilling time & 200 & 200 & 200 & 200 & 200 & - \\
\hline kest_bg & Coefficient in equation for budburst chilling time & 0.1 & 0.1 & 0.1 & 0.1 & 0.1 & - \\
\hline kest_pres & Exponent in equation for budburst chilling time & 1 & 1 & 1 & 1 & 1 & - \\
\hline k_allom1 & Constant in allometry equation & 150 & 150 & 150 & 200 & 200 & - \\
\hline k_allom2 & Constant in allometry equation & 60 & 60 & 60 & 60 & 60 & - \\
\hline
\end{tabular}




\begin{tabular}{|c|c|c|c|c|c|c|c|}
\hline k_allom3 & Constant in allometry equation & 0.67 & 0.67 & 0.67 & 0.67 & 0.67 & - \\
\hline k_chilla & Constant in equation for budburst chilling time & 0 & 0 & 0 & 0 & 0 & - \\
\hline k_chillb & Coefficient in equation for budburst chilling time & 100 & 100 & 100 & 100 & 100 & - \\
\hline k_chilk & Exponent in equation for budburst chilling time & 0.05 & 0.05 & 0.05 & 0.05 & 0.05 & - \\
\hline k_latosa & Tree leaf to sapwood area ratio & 5000 & 5000 & 5000 & 6000 & 6000 & - \\
\hline k_rp & Constant in allometry equation & 1.6 & 1.6 & 1.6 & 1.6 & 1.6 & - \\
\hline leaflong & Leaf longevity (yr) & 3 & 3 & 3 & 0.5 & 0.5 & 1 \\
\hline longevity & Expected longevity under non-stressed conditions (yr) & 500 & 500 & 300 & 400 & 300 & - \\
\hline phengdd5ramp & GDD on $5^{\circ} \mathrm{C}$ base to attain a full leaf cover $\left({ }^{\circ} \mathrm{C}\right.$ day) & 0 & 0 & 0 & 200 & 200 & 100 \\
\hline pstemp_min & Photosynthesis: min. Temperature $\left({ }^{\circ} \mathrm{C}\right)$ & 4 & 4 & 4 & 2 & -4 & -4 \\
\hline pstemp_max & Photosynthesis: max. Temperature $\left({ }^{\circ} \mathrm{C}\right)$ & 38 & 38 & 38 & 38 & 38 & 10 \\
\hline pstemp_low & Low temperature for optimal photosynthesis $\left({ }^{\circ} \mathrm{C}\right)$ & 10 & 10 & 10 & 25 & 10 & 30 \\
\hline pstemp_high & High temperature for optimal photosynthesis $\left({ }^{\circ} \mathrm{C}\right)$ & 15 & 15 & 15 & 25 & 15 & 45 \\
\hline parff_min & Min. forest floor PAR for establishment $\left(\mathrm{J} \mathrm{m}^{-2} \mathrm{day}^{-1}\right)$ & 350 & 2500 & 350 & 350 & 2500 & 1250 \\
\hline respcoeff & Maintenance respiration coefficient & 1 & 1 & 1 & 1 & 1 & 1 \\
\hline rootdist & Fraction of roots in the up soil layer & 0.6 & 0.6 & 0.6 & 0.6 & 0.6 & 0.9 \\
\hline sla & Specific leaf area $\left(\mathrm{m}^{2} \mathrm{~kg}^{-1}\right)$ & 9.3 & 9.3 & 9.3 & 24.3 & 24.3 & 32.4 \\
\hline turnover_leaf & Leaf turnover (fraction $\mathrm{yr}^{-1}$ ) & 0.33 & 0.33 & 0.33 & 1 & 1 & 1 \\
\hline turnover_root & Fine root turnover (fraction $\mathrm{yr}^{-1}$ ) & 0.7 & 0.7 & 0.7 & 0.7 & 0.7 & 0.7 \\
\hline turnover_sap & Sapwood turnover (fraction $\mathrm{yr}^{-1}$ ) & 0.05 & 0.1 & 0.05 & 0.05 & 0.1 & - \\
\hline tcmin_surv & Min. temperature of coldest month for survival & -31 & -31 & -1000 & -14 & -30 & -1000 \\
\hline tcmin_est & Min. temperature of coldest month for establishment $\left({ }^{\circ} \mathrm{C}\right)$ & -30 & -30 & -1000 & -13 & -30 & -1000 \\
\hline
\end{tabular}




\begin{tabular}{|c|c|c|c|c|c|c|c|}
\hline tcmax_est & Max. temperature of coldest month for survival $\left({ }^{\circ} \mathrm{C}\right)$ & -1 & -1 & -2 & 6 & 7 & 1000 \\
\hline twmin_est & Min. temperature of warmest month for survival $\left({ }^{\circ} \mathrm{C}\right)$ & 5 & 5 & -1000 & 5 & -1000 & -1000 \\
\hline woodens & Sapwood and heartwood density $\left(\mathrm{kg} \mathrm{C} \mathrm{m}^{-3}\right)$ & 200 & 200 & 200 & 200 & 200 & - \\
\hline
\end{tabular}


Table S2. The key numerical equations used for the RCA-GUESS coupling processes and the quantification of biogeophysical feedback impacts on vegetation change.

\section{Variables adjusted by LPJ-GUESS}

\begin{tabular}{crr}
\hline Individual LAI: & $L A I=\sum_{n} L A I_{n} \times S_{n}$ & 1.1 \\
\hline Beer's law: & $A_{\text {total }}=2.0-\exp \left(-0.5 \times L A I_{\text {broad-leaved }}\right)-\exp \left(-0.5 \times L A I_{\text {needle-leaved }}\right)$ & 1.2 \\
& $A_{\text {tree }}=\left(1.0-\exp \left(-0.5 \times L A I_{\text {tree }}\right)\right) / A_{\text {total }}$ & 1.3 \\
& $A_{\text {herbaceous }}=1.0-\exp \left(-0.5 \times L A I_{\text {berbaceous }}\right)$ & 1.4 \\
\hline
\end{tabular}

Processes affected in RCA

Surface resistance: $\quad r_{s}=r_{s, \min } \times F_{1} \times{F_{2}}^{-1} \times F_{3}^{-1} \times F_{4}^{-1} \times F_{5}^{-1} / L A I$

Aerodynamic resistance: $\quad r_{a}=1 / g_{b}=1 / f(L A I) \quad 1.6$

Tiled-weighted Albedo: $\quad \alpha_{\text {total }}=\sum\left(A_{n} \times \alpha_{n}\right) / \sum A_{n} \quad 2.0$

Impacts of biophysics to vegetation change

Normalized phenology index: $\quad C_{N P I}=\left(L A I_{e g}-L A I_{d}\right) /\left(L A I_{e g}+L A I_{d}\right) \quad 2.1$

Normalized physiognomy index: $\quad C_{N P M I}=\left(L A I_{w}-L A I_{g}\right) /\left(L A I_{w}+L A I_{g}\right)$

The percentage change for the above indices: $\quad \Delta C=\left(C_{1}-C_{2}\right) \times 100 / 2$

$S:$ phenology state of PFTs.

$A$ : projective vegetation cover.

$r_{s}:$ surface resistance.

$F_{1-5}$ : five influencing factors: the photosynthetically active radiation, the water stress, the vapour pressure deficit, the air temperature dependence and the soil temperature dependence.

$r_{a}$ : aerodynamic resistance based on the conductance $g_{b}$ between the canopy and the canopy air. $f()$ : increasing function (see Samuelsson et al. (2006) for details).

$r_{d}$ : aerodynamic resistance between the canopy floor and the canopy air. $f^{-1}()$ : decreasing function (see Samuelsson et al. (2006) for details).

$\rho$ : air density.

$L_{e}$ : latent heat of vaporisation of water.

$q_{s}$ : surface saturated specific humidity. 
$q_{a m}$ : specific humidity at the first atmospheric level ( $~ 90$ meter).

$T_{s}$ : surface temperature.

$T_{a m}$ : temperature at the first atmospheric level ( $~ 90$ meter).

$e g$ : evergreen PFTs.

$d:$ deciduous PFTs.

$w$ : evergreen PFTs.

$g$ : deciduous PFTs.

Table S3. Aggregation of the International Satellite Land Surface Climatology Project (ISLSCP) II

Potential Natural Vegetation (PNV) Cover dataset and the Kaplan PNV dataset (Kaplan et al., 2003) to five vegetated classes for comparison with plant functional types in RCA-GUESS.

\begin{tabular}{|c|c|c|}
\hline Aggregate class & The validation datasets & RCA-GUESS PFTs \\
\hline Evergreen forests & $\begin{array}{l}\text { Boreal evergreen } \\
\text { Forest/Woodland, } \\
\text { Temperate broad-leaved } \\
\text { evergreen Forest/Woodland, } \\
\text { Temperate needle-leaved } \\
\text { evergreen Forest/Woodland, }\end{array}$ & $\begin{array}{l}\text { BNE-boreal needle-leaved evergreen } \\
\text { trees, } \\
\text { BINE-boreal intolerant needle- } \\
\text { leaved evergreen trees, }\end{array}$ \\
\hline Deciduous forests & $\begin{array}{l}\text { Boreal deciduous } \\
\text { Forest/Woodland }\end{array}$ & $\begin{array}{l}\text { BNS-boreal needle-leaved deciduous } \\
\text { trees, } \\
\text { TBS-temperate deciduous trees, } \\
\text { IBS-temperate intolerant deciduous } \\
\text { trees, }\end{array}$ \\
\hline $\begin{array}{l}\text { Mixed evergreen and } \\
\text { deciduous forests }\end{array}$ & Mixed forests & $\begin{array}{l}\text { Taken from the grid cells other than } \\
\text { the rest classes }\end{array}$ \\
\hline $\begin{array}{l}\text { Herbaceous } \\
\text { vegetation }\end{array}$ & $\begin{array}{l}\text { Dense shrubland, } \\
\text { Open shrubland, } \\
\text { Low- and high-shrub tundra*, } \\
\text { Savanna, Grassland/Steppe }\end{array}$ & GRS-C3 grass \\
\hline Snow and ice & Polar desert/Rock/Ice & None \\
\hline
\end{tabular}

*Kaplan et al, 2003 


\section{References}

Ahlström, A., Schurgers, G., Arneth, A., and Smith, B.: Robustness and uncertainty in terrestrial ecosystem carbon response to CMIP5 climate change projections, Environ. Res. Lett., 7, 044008, doi:10.1088/1748-9326/7/4/044008, 2012b.

Denissenko, E. A., Brovkin, V., and Cramer, W.: NPP Multi-Biome: PIK Data for Northern Eurasia, 1940-1988 (Based on Bazilevich), Data set, available at: http://daac.ornl.gov, from Oak Ridge National Laboratory Distributed Active Archive Center, Oak Ridge, Tennessee, USA, doi:10.3334/ORNLDAAC/575, 2013.

Gower, S.T., Krankina, O., Olson, R. J., Apps, M., Linder, S., and Wang, C.: NPP Boreal Forest: Consistent Worldwide Site Estimates, 1965-1995, R1. Data set, available at: http://daac.ornl.gov] from the Oak Ridge National Laboratory Distributed Active Archive Center, Oak Ridge, Tennessee, USA, doi:10.3334/ORNLDAAC/61, 2012.

Kaplan, J. O., Bigelow, N. H., Prentice, I. C., Harrison, S. P., Bartlein, P. J., Christensen, T. R., Cramer, W., Matveyeva, N. V., McGuire, A. D., Murray, D. F., Razzhivin, V. Y., Smith, B., Walker, D. A., Anderson, P. M., Andreev, A. A., Brubaker, L. B., Edwards, M. E., and Lozhkin, A. V.: Climate change and Arctic ecosystems II: Modeling, paleodata-model com- parisons and future projections, J. Geophys. Res., 108, 8171, doi:10.1029/2002JD002559, 2003.

McGuire, A. D., Christensen, T. R., Hayes, D., Heroult, A., Euskirchen, E., Kimball, J. S., Koven, C., Lafleur, P., Miller, P. A., Oechel, W., Peylin, P., Williams, M., and Yi, Y.: An assessment of the carbon balance of Arctic tundra: comparisons among observations, process models, and atmospheric inversions, Biogeosciences, 9, 3185-3204, doi:10.5194/bg-9-3185-2012, 2012.

Olson, R. J., Scurlock, J. M. O., Prince, S. D., Zheng, D. L., and Johnson, K. R (Eds.): NPP MultiBiome: NPP and Driver Data for Ecosystem Model Data Intercomparison, R2. Data set, available at: http://daac.ornl.gov, from Oak Ridge National Laboratory Distributed Active Archive Center, Oak Ridge, Tennessee, USA, doi:10.3334/ORNLDAAC/615, 2013a.

Olson, R. J., Scurlock, J. M. O., Prince, S. D., Zheng, D. L., and Johnson, K. R. (Eds.): NPP MultiBiome: Global Primary Production Data Initiative Products, R2. Data set, available at: http://daac.ornl.gov, from the Oak Ridge National Laboratory Distributed Active Archive Center, Oak Ridge, Tennessee, USA, doi:10.3334/ORNLDAAC/617, 2013 b. 
Samuelsson, P., Gollvik, S., and Ullerstig, A.: The land-surface scheme of the Rossby Centre regional atmospheric climate model (RCA3), Reports Meteorol. Climatol., 12, p. 38, 2006.

Zheng, D. L., Prince, S. D., and Wright, R.: NPP Multi-Biome: Gridded Estimates for Selected RegionsWorldwide, 1954-1998, R3. Data set, available at: http://daac.ornl.gov, from the Oak Ridge National Laboratory Distributed Active Archive Center, Oak Ridge, Tennessee, USA, doi:10.3334/ORNLDAAC/614, 2013. 